http://jmscr.igmpublication.org/home/ ISSN (e)-2347-176x ISSN (p) 2455-0450

crossref DOI: https://dx.doi.org/10.18535/jmscr/v8i2.80

\title{
Efficacy of Concurrent Chemoradiation in Locally Advanced Squamous Cell Carcinoma of Head and Neck
}

\author{
Authors \\ L.Karpagavalli $^{1 *}$, Joneetha Jones ${ }^{2}$, K.Sureshkumar ${ }^{3}$ \\ ${ }^{1}$ Assistant Professor, Dept of Radiotherapy, Tamilnadu Government Multi Super Speciality Hospital, \\ Chennai, Tamil Nadu, India \\ ${ }^{2}$ Assistant Professor, Dept of Radiotherapy, Malabar Cancer Centre, Thalassery, Kerala, India \\ ${ }^{3}$ Additional Professor, Dept of Radiotherapy, Government Medical College, Kottayam, Kerala, India \\ *Corresponding Author \\ Dr L.Karpagavalli
}

\begin{abstract}
Aims \& Objectives: To analyse the loco regional control and acute toxicity in patients with locally advanced squamous cell carcinoma of the head and neck (oropharynx and larynx) in Conventional concurrent radiotherapy with weekly Cisplatin.

Materials and Methodology: A single arm prospective study of 30 consecutive cases of newly diagnosed patients with locolly advanced Stage III \& IVA Squamous cell carcinoma of oropharynx \& larynx enrolled .All patients were irradiated with conventional fractionation to a dose of 66 Gy in 33 fractions over 6.5-7 weeks along with weekly Inj. Cisplatin $40 \mathrm{mg} /$. The immediate locoregional response rates were assessed clinically and toxicity profile of the treatment was assessed with RTOG acute morbidity scoring criteria.The study was statistically analysed using Chi Square test.

Results: Out of thirty patients, seven percent (7\%) have undergone tracheostomy. Out of thirty patients, eighty three (83\%) were having complete response and seventeen percent (17\%) were having partial response. All the patients experienced at least grade 1 skin reaction after $40-45$ Gy. No grade 4 skin reactions were seen. Twenty three i.e. $77 \%$ patients had grade 2 patchy mucositis and 5 had grade 3 confluent mucositis.

Conclusion: The present schedule is well tolerated for all patients and can be safely used in older patients and patients with controlled co-morbidities. This conventional fractionation schedule suits the busy and heavy patient load in our institute and is highly cost effective.
\end{abstract}

Keywords: concurrent chemoradiation, weekly cisplatin, locoregional control, mucositis, skin reaction.

\section{Introduction}

Cancers of the head and neck arise from the lining membrane of the upper aerodigestive $\operatorname{tract}^{1}$. It usually refers to epithelial neoplasm arising from below the skull base to the region of thoracic inlet. They are a diverse group of diseases each with distinct epidemiologic, anatomic and pathologic features. They show wide variation in natural history, prognosis and treatment considerations.

Head and neck cancer is an area of great importance to the researchers and oncologists because of the physical and psychological morbidity it produces. It also causes significant burden on the family and society. 
India is one of the high incidence zones for head \& neck cancer. In India, the most common H\&N cancers are those of oral cavity and pharynx. Men are more affected than women. Age adjusted incidence for these sites in Indian males range from 10.8 to 38.8 and among females, 6 to 15 per 1 lakh population. In fact, oral cavity and pharynx cancers are 3rd most common cancer in males and 4th most common in females in the developing countries. Overall there is evidence that the toxicities of therapy administered for these malignancies diminish over time, and overall quality of life improves with treatment of the disease. It remains to be seen how quality of life measurements and analysis will affect an individual patient, as these instruments are being integrated into research efforts on population based samples ${ }^{45}$.

Wodinsky et al in 1974 showed the radio sensitising effect of Cisplatin on experimental tumors. Cisplatin given before irradiation causes an increase in slope of radiation dose response curve. The exact mechanism of action is not known. Preliminary experiments show it ability to inhibit DNA synthesis and to a lesser extent, RNA and Protein synthesis. It binds to DNA and forms inter- and intra-strand DNA adducts. Cisplatin inhibits sublethal and potentially lethal damage repair. Antitumor activity is greater if administered by continuous infusion because it may be phase and cycle nonspecific drug with preferential action on G1 phase of cell cycle.

Recent advances in translational and clinical research have led to a paradigm shift wherein radical radiotherapy with concurrent chemotherapy (3-weekly high-dose cisplatin) is now considered the contemporary standard of care in the nonsurgical management of loco-regionally advanced SCCHN (MACH-NC) update also states that combined concomitant CT-RT is the standard treatment in nonresectable patients, and is also preferable in resectable patients, when the anticipated functional outcome with surgery is poor.

\section{Aim \& Objectives}

The aim of this study was to analyse the efficacy of concurrent chemoradiation in locally advanced squamous cell carcinoma of head and neck in terms of locoregional control and toxicity.

\section{Materials and Methods \\ Study Design}

The study was a single arm prospective study with 30 consecutive patients who fulfilled the inclusion criteria were enrolled.

\section{Inclusion Criteria}

- Patients with histological proof (from the primary lesion and/or lymph nodes) of squamous cell carcinoma of the oropharynx.

- Patients with Stage III or IVA disease (M0) (AJCC-UICC $7^{\text {th }}$ edition)

- Age less than 65 years.

- Patients with a Zubrod/ECOG performance status of $0,1,2$.

- No distant metastatic disease.

- Patients with adequate bone marrow function and normal renal function test

- No symptomatic coronary artery disease (angina) or myocardial infarction within the last 6 months.

\section{Exclusion Criteria}

- Histology other than squamous cell carcinoma.

- Evidence of metastases

- Prior chemotherapy for any reason or prior radiotherapy to the head and neck region.

- Initial surgical treatment excluding diagnostic biopsy of the primary site or neck disease.

- Patients having tracheostomy.

- Patients with simultaneous primaries.

Pregnant women because of the embryo toxic effects of chemotherapy.

\section{Radiation schedule and Chemo dosage}

All patients are treated with external beam radiation 66 Gy in 2Gy per fraction with 5 fractions per week with concurrent chemo Inj. Cisplatin $40 \mathrm{mg} / \mathrm{m} 2$ every week. 


\section{Toxicity Assessment}

Patients were seen daily during the treatment and complaints were attended to. Endoscopic evaluation and biopsy was mandatory before treatment. Similarly chest x-ray, complete blood count, renal and liver function tests and dental evaluation was compulsory. History/physical exam, weight, hemogram, renal function, tumor measurement and toxicity evaluation was done every week during radiotherapy and at six weeks.

\section{Follow up and Response Evaluation}

All patients were reassessed by clinical examination and with a CECT Neck, 6 weeks after completion of concurrent chemo radiation. Response to treatment was described based on the

Response Evaluation Criteria in Solid Tumors (RECIST 1.1 version) Criteria.

\section{Statistical Analysis}

The $\mathrm{p}$ value was assessed using Chi square test.

\section{Results}

Of the 30 patients, thirty seven percentage (37\%) were having base of tongue as primary site, $23 \%$ in supraglottic larynx, 20\% in tonsillar region, and only one patient $(3.3 \%)$ was having primary in subglottic larynx.

\section{Grade of the tumour}

Of the 30 patients, $8(27 \%)$ had well differentiated tumors; $10(33 \%)$ had moderately differentiated tumors and $12(40 \%)$ had poorly differentiated tumors.

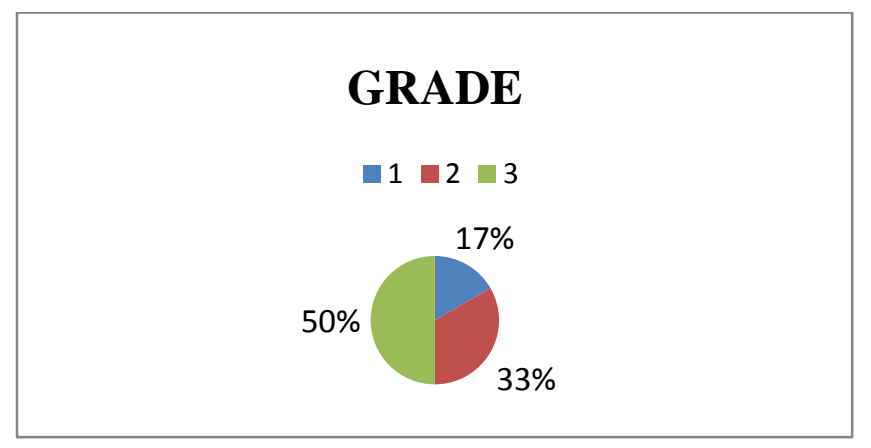

\section{Stage grouping}

Fig No: 1

Seventeen $(57 \%)$ patients had stage III disease and thirteen $(43 \%)$ patients had stage IVA disease (AJCC 2007).

\section{STAGE GROUPING}

$\square$ STAGE III $\square$ STAGE IVA

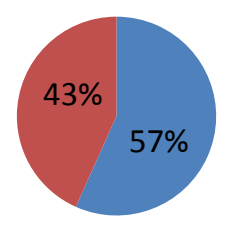

Fig No: 2

Complete Response

\begin{tabular}{|l|c|c|}
\hline & Number Of Patients & Percentage \\
\hline $\begin{array}{l}\text { Complete } \\
\text { Response }\end{array}$ & 25 & 83 \\
\hline $\begin{array}{l}\text { Partial } \\
\text { Response }\end{array}$ & 5 & 17 \\
\hline & 30 & 100 \\
\hline
\end{tabular}

Out of thirty patients, eighty three (83\%) were having complete response and seventeen percent $(17 \%)$ were having partial response.

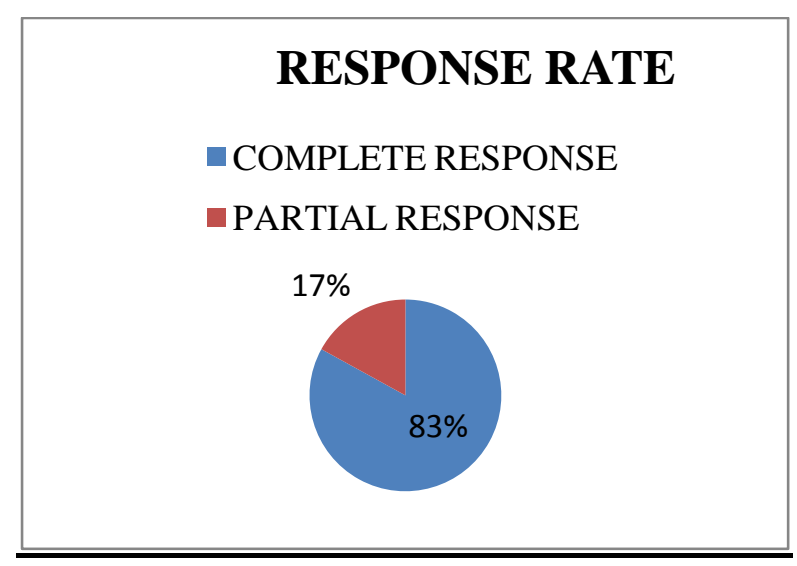

\section{Event}

Fig No: 3

Out of partial responders, seven percent $(7 \%)$ had primary failure, seven percent $(7 \%)$ had nodal failure, three percentage of patients had primary and nodal failure

\section{Toxicity Assessment}

All the patients experienced at least grade 1 skin reaction in the form of dry desquamation and epilation. It was seen after $40-45$ Gy. No grade 4 skin reactions were seen. Twenty three i.e. $77 \%$ patients had grade 2 patchy mucositis with moderate pain requiring analgesics and 5 had grade 3 confluent mucositis requiring narcotic analgesics. 
These grade 3 reactions were seen only near the end of treatment with minimal treatment interruption.

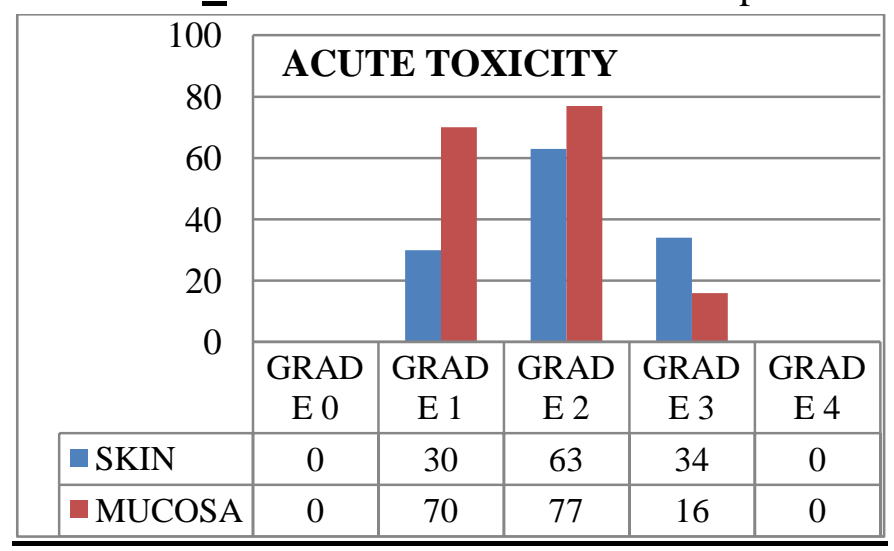

Fig No: 4

\section{Impact of Locoregional Control and Disease Free Survival}

Patients who had complete response having six month period of disease free survival, and in partial responders disease free interval is less than six months. $\mathrm{p}-0.000$ (significant)

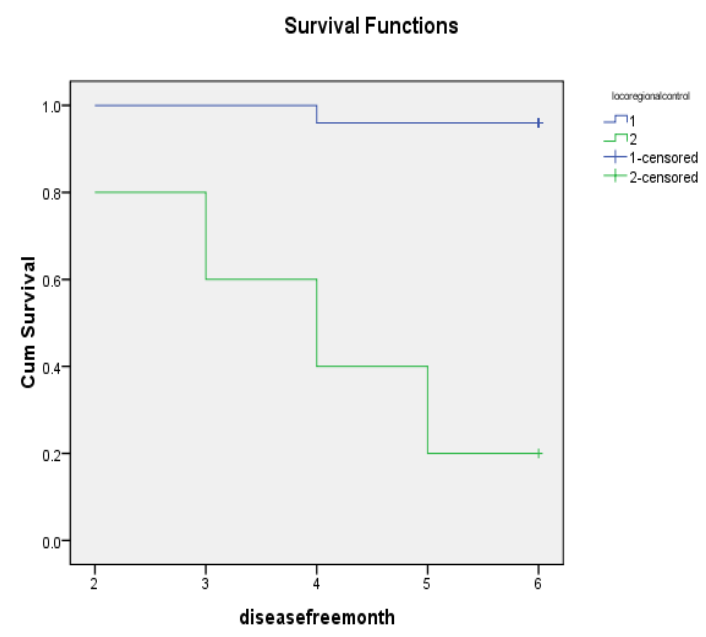

Fig No: 5

\section{Discussion}

Despite advances in the understanding of the biology and pathogenesis of head and neck cancer (HNC), and despite improvements in imaging modalities, locoregionally advanced (stage III and stage IV) squamous cell carcinoma of the head and neck remains a difficult management problem. The standard of care for resectable squamous cell carcinoma of the head and neck has traditionally been surgical excision followed by radiotherapy.
Despite advances in surgical techniques, surgical excision followed by radiotherapy may lead to multiple problems with function and/or cosmesis. Attempts at using surgery as a radiation sparing modality_are limited since most patients with stage III and stage IV head and neck cancer $r$ require radiotherapy postoperatively.

Unresectable head and neck cancer generally implies biologically and anatomically more aggressive disease with a smaller chance for permanent eradication of the disease. It is difficult, however, to find a precise definition of resectability for these tumors. The limits of respectability vary between surgeons, institutions, and patients. Likewise, the acceptability of a near complete excision may alter the definition of resectability. Large tumors of the oral tongue or base of tongue may be "resectable", but require a total glossectomy. While this may be technically possible, this is unacceptable to many patients.

The meta-analysis (MACH-NC) also allowed a new comparison of the benefit associated with concomitant versus induction chemotherapy. It is interesting to note that both the indirect and the direct comparisons were consistent on survival, event-free survival and loco-regional failure, showing a clear advantage in favour of concomitant chemotherapy

\section{Conclusion}

In conclusion, this study shows that Chemoradiation with weekly Cisplatin and conventional RT achieves local control rates equal to other more toxic altered fractionation and high dose chemotherapy schedules in literature. The present schedule is well tolerated for all patients and can be safely used in older patients and patients with controlled co-morbidities. This conventional fractionation schedule suits the busy and heavy patient load in our institute and is highly cost effective. There is a need for further studies with large number of patients with head to head randomization of various dose schedules and longer follow-up for survival analysis and to choose optimal dose and duration of chemotherapy. 


\section{References}

1. Beckmann GK, Hoppe F, Pfreundner L, Flentje MP. (2005) Hyperfractionated accelerated radiotherapy in combination with weekly cisplatin for locally advanced head and neck cancer. Head Neck 27: 36-43, 2005.

2. Jose Antonio Medina, Antonio Rueda, et al: A phase II study of concomitant boost radiation plus concurrent weekly cisplatin for locally advanced unresectable head and neck carcinomas. Radiotherapy and Oncology: 34-38, 2006.

3. Brizel D M (2007)The Role of Combined Radiotherapy and Chemotherapy in the Management of Locally Advanced Squamous Carcinoma of the Head and Neck In: Halprein E C, Perez C A, Brady L W, (eds.) Principles and Practice of Radiation Oncology, $5^{\text {th }}$ edn.Philadelphia, Wolters Kluwer Pp 807-19.

4. Shrivastava S K, Sarin R, et al. (2011) Evidence Based Management Guidelines Vol. IV Mumbai The Tata Memorial Hospital.

5. Pignon JP, Bourhis J, Domenge $\mathrm{C}$, et al: Chemotherapy added to locoregional treatment for head and neck squamous-cell carcinoma: Three meta-analyses of updated individual data-MACH-NC Collaborative Group, MetaAnalysis of Chemotherapy on Head and Neck Cancer. Lancet 355:949-955, 2000

6. Bourhis J, Amand C, Pignon J P, on behalf of the MACH-NC Collaborative Group (2004) Update of MACH-NC (Meta-Analysis of Chemotherapy in Head \& Neck Cancer) database focused on concomitant chemoradiotherapy. Journal of Clinical Oncology, 2004 ASCO Annual Meeting Proceedings (Post-Meeting Edition). Vol 22, No 14S (July 15 Supplement), 2004: 5505

7. Jean-Pierre Pignon, Aurélie le Maître, Emilie Maillard, Jean Bourhis, on behalf of the MACH-NC Collaborative Group (2009) Update of MACH-NC (Meta-Analysis of Chemotherapy in Head \& Neck Cancer) database focused on concomitant chemoradiotherapy. Radiotherapy and Oncology Vol 92, 4 -14 (2009).

8. Tanguy Y Seiwert, Joseph K Salama and Everett E Vokes (2007) The Concurrent Chemoradiation Paradigm-General Principles. Nature Clinical Practice Oncology February 2007 Vol 4 No 2, Pp 86-100.

9. Steel GG and Peckham MJ (1979) Exploitable mechanisms in combined radiotherapy chemotherapy: the concept of additivity. Int J Radiat Oncol Biol Phys 5: 85-91

10. Brizel D M, Esclamado R, (2006) Concurrent Chemoradiotherapy for Locally Advanced, Non-metastatic, Squamous Carcinoma of the Head and Neck: Consensus, Controversy, and Conundrum. Journal of clinical oncology, volume 24 number 17 June 2006. Pp 2612-17.

11. Page D L, et al. (eds) AJCC Cancer Staging Manual $7^{\text {th }}$ edn, NY. Springer. 